\title{
Multiple Gamma Function and Its Application to Computation of Series
}

\author{
V. S. Adamchik \\ Carnegie Mellon University, \\ Pittsburgh, USA
}

\begin{abstract}
The multiple gamma function $\Gamma_{n}$, defined by a recurrence-functional equation as a generalization of the Euler gamma function, was originally introduced by Kinkelin, Glaisher, and Barnes around 1900. Today, due to the pioneer work of Conrey, Katz and Sarnak, interest in the multiple gamma function has been revived. This paper discusses some theoretical aspects of the $\Gamma_{n}$ function and their applications to summation of series and infinite products.
\end{abstract}

Keywords: multiple gamma function, Barnes function, gamma function, Riemann zeta function, Hurwitz zeta function, Stirling numbers, Stieltjes constants, Catalan's constant, harmonic numbers, Glaisher's constant

2000 Mathematics Classification: Primary 33E20, 33F99, 11M35, 11B73

\section{Introduction}

The Hurwitz zeta function, one of the fundamental transcendental functions, is traditionally defined (see [1] ) by the series

$$
\zeta(s, z)=\sum_{k=0}^{\infty} \frac{1}{(k+z)^{s}}, \quad \Re(s)>0 .
$$

It admits an analytic continuation to the entire complex plane except for the simple pole $s=1$. The Riemann zeta function $\zeta(s)$ is a special case of $\zeta(s, z)$

$$
\zeta(s, 1)=\zeta(s)
$$

The Hurwitz function has quite a few series and integral representations (see [1, 8]). The most famous is the Hermite integral:

$$
\begin{gathered}
\zeta(s, z)=\frac{z^{-s}}{2}+\frac{z^{1-s}}{s-1}+2 \int_{0}^{\infty} \frac{\sin \left(s \arctan \left(\frac{x}{z}\right)\right)}{\left(x^{2}+z^{2}\right)^{s / 2}\left(e^{2 \pi x}-1\right)} d x, \\
s \neq 1, \Re(z)>0
\end{gathered}
$$

(c) 2008 Kluwer Academic Publishers. Printed in the Netherlands. 
from which one can deduce many fundamental properties of the Hurwitz function, including the asymptotic expansion at infinity:

$\zeta(s, z)=\frac{z^{1-s}}{s-1}+\frac{z^{-s}}{2}+\sum_{j=1}^{m-1} \frac{B_{2 j} \Gamma(2 j+s-1)}{(2 j) ! \Gamma(s)} z^{-2 j-s+1}+O\left(\frac{1}{z^{2 m+s+1}}\right)$

The Hurwitz function is closely related to the multiple gamma function $\Gamma_{n}(z)$ defined as a generalization of the classical Euler gamma function $\Gamma(z)$, by the following recurrence-functional equation (for references and a short historical survey see [6, 19]):

$$
\begin{aligned}
\Gamma_{n+1}(z+1) & =\frac{\Gamma_{n+1}(z)}{\Gamma_{n}(z)}, \quad z \in \mathbb{C}, \quad n \in \mathbb{N} \\
\Gamma_{1}(z) & =\Gamma(z) \\
\Gamma_{n}(1) & =1 .
\end{aligned}
$$

The multiple gamma function, originally introduced over 100 years ago, has significant applications in the connection with the Riemann Hypothesis. Montgomery [14] and Sarnak [17] have conjectured that the limiting distribution of the non-trivial zeros of the Riemann zeta function is the same as that of the eigenphases of matrices in the CUE (the circular unitary ensemble). It has been shown in works by Mehta, Sarnak, Conrey, Keating, and Snaith that a closed representation for statistical averages over CUE of $N \times N$ unitary matrices, when $N \rightarrow \infty$ can be expressed in terms of the Barnes function $G(z)=1 / \Gamma_{2}(z)$, defined by

$$
\begin{aligned}
G(z+1) & =G(z) \Gamma(z), \quad z \in \mathbb{C} \\
G(1) & =1 .
\end{aligned}
$$

Keating and Snaith 9, 10] conjectured the following relationship between the moments of $\left|\zeta\left(\frac{1}{2}+i t\right)\right|$, averaged over $t$, and characteristic polynomials, averaged over the CUE:

$$
\begin{gathered}
\log \left(\frac{1}{a(\lambda)} \lim _{T \rightarrow \infty} \frac{1}{\log ^{\lambda^{2}}(T) T} \int_{0}^{T}\left|\zeta\left(\frac{1}{2}+i t\right)\right|^{2 \lambda} d t\right)= \\
\lambda^{2}(\gamma+1)-2 \lambda \sum_{k=2}^{\infty}(-\lambda)^{k}\left(2^{k}-1\right) \frac{\zeta(k)}{k+1},
\end{gathered}
$$

where $a(\lambda)$ is a known function of $\lambda \in \mathbb{N}$. The series on the right hand side of (5) is understood in a sense of analytic continuation (see [1] 
for the method of evaluation of such sums), provided by the Barnes function

$$
\begin{array}{r}
2 \sum_{k=2}^{\infty}(-z)^{k} \frac{\zeta(k)}{k+1}=\frac{2}{z} \log G(z+1)+z(\gamma+1)-\log (2 \pi)+1 \\
|z|<1
\end{array}
$$

where $\gamma$ denotes the Euler-Mascheroni constant. Conversely, we find that the moments of $\left|\zeta\left(\frac{1}{2}+i t\right)\right|$ in (5) are just a ratio of two Barnes functions:

$$
\log \left(\frac{1}{a(\lambda)} \lim _{T \rightarrow \infty} \frac{1}{\log ^{\lambda^{2}}(T) T} \int_{0}^{T}\left|\zeta\left(\frac{1}{2}+i t\right)\right|^{2 \lambda} d t\right)=\frac{G(\lambda+1)^{2}}{G(2 \lambda+1)}
$$

The evidence in support of Keating and Snaith's conjecture is confirmed by a few particular cases $\lambda=1,2,3,4$, and the numerical experiment, conducted by Odlyzko [15], for $T$ up to $10^{22}$ th zero of the Riemann zeta function.

The identity ([6) can be further generalized to

$$
\sum_{k=2}^{\infty}(-z)^{k} \frac{\zeta(k)}{k+r-1}, \quad r \in \mathbb{Q}, \quad|z|<1
$$

and then evaluated in terms of the $r$-tiple gamma functions. For instance, with $r=3$ we find

$$
\begin{gathered}
\sum_{k=2}^{\infty}(-z)^{k} \frac{\zeta(k)}{k+2}=\frac{2}{z^{2}} \log \Gamma_{3}(z+1)+\frac{1}{z^{2}} \log G(z+1)+ \\
\frac{6 z^{2}+3 z-1}{12 z}+\frac{\gamma z}{3}-\frac{\log (2 \pi)}{2}-\frac{2 \zeta^{\prime}(-1)}{z}, \quad|z|<1
\end{gathered}
$$

In this paper we aim at developing a mathematical foundation for symbolic computation of special classes of infinite series and products. Generally speaking, all series (subject to convergence) of the form:

$$
\sum_{j=1}^{\infty} R(j) \log ^{m} P(j), \quad m \in \mathbb{N}
$$

where $R(z)$ and $P(z)$ are polynomials, can be expressed in a closed form by means of the multiple gamma function, which may further simplify to elementary functions. This algorithm also complements the 
work previously started by Adamchik and Srivastava [1] for symbolic summation of series involving the Riemann zeta function. The algorithm will expand the capabilities of existing software packages for non-hypergeometric summation.

\section{Asymptotics of $\zeta^{\prime}(-\lambda, z)$}

In this section, based on the integral (2), we derive the asymptotic expansion of $\zeta^{\prime}(t, z)=\frac{d}{d t} \zeta(t, z)$, when $t=-\lambda, \lambda \in N_{0}$ and $z \rightarrow \infty$. Differentiating both sides of (2) with respect to $s$, we obtain

$$
\begin{aligned}
\zeta^{\prime}(-\lambda, z) & =\frac{z^{\lambda+1}}{\lambda+1} \log z-\frac{z^{\lambda}}{2} \log z-\frac{z^{\lambda+1}}{(\lambda+1)^{2}} \\
& +2 \int_{0}^{\infty} \frac{\tan ^{-1}\left(\frac{x}{z}\right) \cos \left(\lambda \tan ^{-1}\left(\frac{x}{z}\right)\right)}{\left(e^{2 \pi x}-1\right)\left(x^{2}+z^{2}\right)^{-\lambda / 2}} d x \\
& +\int_{0}^{\infty} \frac{\log \left(x^{2}+z^{2}\right) \sin \left(\lambda \tan ^{-1}\left(\frac{x}{z}\right)\right)}{\left(e^{2 \pi x}-1\right)\left(x^{2}+z^{2}\right)^{-\lambda / 2}} d x
\end{aligned}
$$

Next, we expand the integrands into the Taylor series with respect to $z$. Taking into account that

$$
\left(x^{2}+z^{2}\right)^{\lambda / 2} \cos \left(\lambda \tan ^{-1}\left(\frac{x}{z}\right)\right)=\sum_{k=0}^{\lambda / 2}(-1)^{k}\left(\begin{array}{c}
\lambda \\
2 k
\end{array}\right) z^{\lambda-2 k} x^{2 k}
$$

and

$$
\left(x^{2}+z^{2}\right)^{\lambda / 2} \sin \left(\lambda \tan ^{-1}\left(\frac{x}{z}\right)\right)=\sum_{k=0}^{\lambda / 2}(-1)^{k}\left(\begin{array}{c}
\lambda \\
2 k+1
\end{array}\right) z^{\lambda-2 k-1} x^{2 k+1}
$$

we compute

$$
\begin{gathered}
\int_{0}^{\infty} \frac{\tan ^{-1}\left(\frac{x}{z}\right) \cos \left(\lambda \tan ^{-1}\left(\frac{x}{z}\right)\right)}{\left(e^{2 \pi x}-1\right)\left(x^{2}+z^{2}\right)^{-\lambda / 2}} d x= \\
\sum_{k=0}^{\lambda / 2} z^{\lambda-2 k}(-1)^{k}\left(\begin{array}{c}
\lambda \\
2 k
\end{array}\right) \int_{0}^{\infty} \frac{x^{2 k} \tan ^{-1}\left(\frac{x}{z}\right)}{e^{2 \pi x}-1} d x
\end{gathered}
$$

and 


$$
\begin{gathered}
\int_{0}^{\infty} \frac{\log \left(x^{2}+z^{2}\right) \sin \left(\lambda \tan ^{-1}\left(\frac{x}{z}\right)\right)}{\left(e^{2 \pi x}-1\right)\left(x^{2}+z^{2}\right)^{-\lambda / 2}} d x= \\
-\sum_{k=0}^{\lambda / 2} z^{-2 k+\lambda-1}(-1)^{k}\left(\begin{array}{c}
\lambda \\
2 k+1
\end{array}\right) \int_{0}^{\infty} \frac{x^{2 k+1} \log \left(x^{2}+z^{2}\right)}{e^{2 \pi x}-1} d x .
\end{gathered}
$$

In the next step, we find asymptotic expansions of integrals on the right hand side of (9) and (10) when $z \rightarrow \infty$. Expanding $\tan ^{-1}\left(\frac{x}{z}\right)$ and $\log \left(x^{2}+z^{2}\right)$ into the Taylor series with respect to $x$ and performing term by term integration, we arrive at

$$
\begin{gathered}
\int_{0}^{\infty} \frac{x^{2 k} \tan ^{-1}\left(\frac{x}{z}\right)}{e^{2 \pi x}-1} d x=\sum_{j=0}^{r} \frac{(-1)^{k} z^{-2 j-1} B_{2(j+k+1)}}{4(2 j+1)(j+k+1)}+O\left(\frac{1}{z^{2 r+3}}\right), \\
\int_{0}^{\infty} \frac{x^{2 k+1} \log \left(1+x^{2} / z^{2}\right)}{e^{2 \pi x}-1} d x=-\sum_{j=1}^{r} \frac{(-1)^{k} z^{-2 j} B_{2(j+k+1)}}{4 j(j+k+1)}+O\left(\frac{1}{z^{2 r+2}}\right),
\end{gathered}
$$

where $B_{k}$ are the Bernoulli numbers. Substituting these into (9) and (10) and the latters into (8), after some tedious algebra, we obtain

PROPOSITION 1. The derivatives of the Hurwitz zeta function have the following asymptotic expansion when $z \rightarrow \infty$ :

$$
\begin{aligned}
\zeta^{\prime}(-\lambda, z) & =\frac{z^{\lambda+1}}{\lambda+1} \log z-\frac{z^{\lambda}}{2} \log z-\frac{z^{\lambda+1}}{(\lambda+1)^{2}} \\
& +\sum_{j=1}^{r} \frac{z^{-2 j+\lambda+1}}{(2 j) !} B_{2 j} \sum_{k=0}^{2 j-1}(k+1)\left[\begin{array}{c}
2 j-1 \\
k+1
\end{array}\right](-\lambda)^{k} \\
& -\log z \sum_{j=1}^{r} \frac{z^{-2 j+\lambda+1} B_{2 j}(-\lambda)_{2 j-1}}{(2 j) !}+O\left(\frac{1}{z^{2 r-\lambda+1}}\right)
\end{aligned}
$$

where $\zeta^{\prime}(t, z)=\frac{d}{d t} \zeta(t, z),(-\lambda)_{k}=(-\lambda)(-\lambda+1) \ldots(-\lambda+k-1)$ is the Pochhammer symbol, and $\left[\begin{array}{l}n \\ k\end{array}\right]$ are the Stirling cycle numbers, defined recursively [16] by

$$
\left[\begin{array}{l}
n \\
k
\end{array}\right]=(n-1)\left[\begin{array}{c}
n-1 \\
k
\end{array}\right]+\left[\begin{array}{l}
n-1 \\
k-1
\end{array}\right], \quad\left[\begin{array}{l}
n \\
0
\end{array}\right]=\left\{\begin{array}{cc}
1, & n=0 \\
0, & n \neq 0
\end{array}\right.
$$


For $\lambda=0$ and $\lambda=1$, formula (11) yields the well-know asymptotics 11]:

$$
\begin{aligned}
& \zeta^{\prime}(0, N)=N \log N-\frac{\log N}{2}-N+O\left(\frac{1}{N}\right) \\
& \zeta^{\prime}(-1, N)=\left(\frac{N^{2}}{2}-\frac{N}{2}+\frac{1}{12}\right) \log N-\frac{N^{2}}{4}+\frac{1}{12}+O\left(\frac{1}{N^{2}}\right)
\end{aligned}
$$

In the similar way, we can derive the asymptotic expansions for higher order derivatives $\zeta^{(m)}(-\lambda, N)$, where $N \rightarrow \infty$ and $\lambda \in \mathbb{N}^{+}$.

\section{Multiple gamma, zeta and the Hurwitz functions}

In [20], Vardi expressed the $\Gamma_{n}$ function in terms of the multiple zeta function $\zeta_{n}(s, z)$, as

$$
(-1)^{n} \log \Gamma_{n}(z)=-\lim _{s \rightarrow 0}\left(\frac{\partial \zeta_{n}(s, z)}{\partial s}\right)-\sum_{k=1}^{n}(-1)^{k}\left(\begin{array}{c}
z \\
k-1
\end{array}\right) R_{n+1-k}
$$

where

$$
R_{n}=\sum_{k=1}^{n} \lim _{s \rightarrow 0}\left(\frac{\partial \zeta_{k}(s, 1)}{\partial s}\right)
$$

and

$$
\begin{aligned}
\zeta_{n}(s, z) & =\sum_{k_{1}=0}^{\infty} \ldots \sum_{k_{n}=0}^{\infty} \frac{1}{\left(k_{1}+k_{2}+\ldots+k_{n}+z\right)^{s}} \\
& =\sum_{k=0}^{\infty} \frac{1}{(k+z)^{s}}\left(\begin{array}{c}
k+n-1 \\
n-1
\end{array}\right)
\end{aligned}
$$

The aim of this section is to find a closed form representation for $\log \Gamma_{n}(z)$ in finite terms of the Hurwitz zeta function, and vice versa.

PROPOSITION 2. The multiple gamma function $\Gamma_{n}(z)$ may be expressed by means of the derivatives of the Hurwitz zeta function:

$$
\log \Gamma_{n}(z)=\frac{(-1)^{n}}{(n-1) !} \sum_{k=0}^{n-1} P_{k, n}(z)\left(\zeta^{\prime}(-k)-\zeta^{\prime}(-k, z)\right), \quad \Re(z)>0
$$


where the polynomials $P_{k, n}(z)$ are defined by

$$
P_{k, n}(z)=\sum_{j=k+1}^{n}(-z)^{j-k-1}\left(\begin{array}{c}
j-1 \\
k
\end{array}\right)\left[\begin{array}{l}
n \\
j
\end{array}\right]
$$

where $\left[\begin{array}{l}n \\ j\end{array}\right]$ are the Stirling cycle numbers.

The polynomials $P_{k, n}(z)$ can be envisaged as the generalized Stirling polynomials of the first kind, generated by

$$
\prod_{k=1}^{n-1}(k+x-z)=\sum_{k=0}^{n-1} P_{k, n}(z) x^{k}
$$

with the following two alternative forms of representation

$$
P_{k, n}(z)=\left.\frac{(-1)^{k}}{k !} \frac{\partial^{n-1}}{\partial y^{n-1}} \frac{\log ^{k}(1-y)}{(1-y)^{1-z}}\right|_{y \rightarrow 0}
$$

and

$$
P_{k, n}(z)=\sum_{i=k+1}^{n}\left(\begin{array}{c}
z \\
n-i
\end{array}\right) \frac{(n-1) !}{(i-1) !}\left[\begin{array}{c}
i \\
k+1
\end{array}\right]
$$

For $z=1$ the polynomials $P_{k, n}(z)$ are simplified to the Stirling numbers:

$$
P_{k, n}(1)=\left[\begin{array}{c}
n-1 \\
k
\end{array}\right]
$$

Interestingly, the polynomials $P_{k, n}(z)$ were first considered by Mitrinovic [13 40 years ago (with no relation to the multiple gamma function) as a possible generalization of the Stirling numbers.

The proof of Proposition 2 is based on the following two lemmas.

LEMMA 1. The multiple zeta function $\zeta_{n}(s, z)$ defined by (16) may be expressed by means of the Hurwitz function

$$
\zeta_{n}(s, z)=\frac{1}{(n-1) !} \sum_{j=0}^{n-1} P_{j, n}(z) \zeta(s-j, z)
$$

Proof. Recall the definition of the Stirling cycle numbers

$$
\left(\begin{array}{c}
k+n-1 \\
n-1
\end{array}\right)=\frac{1}{(n-1) !} \sum_{i=0}^{n} k^{i-1}\left[\begin{array}{c}
n \\
i
\end{array}\right]
$$


Expanding $k^{i-1}=((k+z)-z)^{i-1}$ by the binomial theorem, implies that

$$
\left(\begin{array}{c}
k+n-1 \\
n-1
\end{array}\right)=\frac{1}{(n-1) !} \sum_{i=0}^{n} \sum_{j=0}^{i-1}\left(\begin{array}{c}
i-1 \\
j
\end{array}\right)(k+z)^{j}(-z)^{i-j-1}\left[\begin{array}{c}
n \\
i
\end{array}\right]
$$

Interchanging the order of summation and making use of (18), leads us to

$$
\left(\begin{array}{c}
k+n-1 \\
n-1
\end{array}\right)=\frac{1}{(n-1) !} \sum_{j=0}^{n} P_{j, n}(z)(k+z)^{j}
$$

Finally, we substitute this binomial coefficients representation into (16):

$$
\begin{aligned}
\zeta_{n}(s, z) & =\sum_{k=0}^{\infty} \frac{1}{(k+z)^{s}}\left(\begin{array}{c}
k+n-1 \\
n-1
\end{array}\right) \\
& =\frac{1}{(n-1) !} \sum_{j=0}^{n} P_{j, n}(z) \sum_{k=0}^{\infty} \frac{1}{(k+z)^{s-j}}
\end{aligned}
$$

We complete the proof by evaluating the inner sum to the Hurwitz zeta function.

LEMMA 2. The function $R_{n}$ defined by (15) may be expressed by means of the derivatives of the Riemann zeta function

$$
R_{n}=\frac{1}{(n-1) !} \sum_{k=0}^{n-1} \zeta^{\prime}(-k)\left[\begin{array}{c}
n \\
k+1
\end{array}\right]
$$

Proof. Using Lemma 1 with $z=1$ and formula (21), we get

$$
\zeta_{k}(s, 1)=\frac{1}{(k-1) !} \sum_{j=0}^{k}\left[\begin{array}{c}
k-1 \\
j
\end{array}\right] \zeta(s-j)
$$

which, upon differentiation, yields

$$
\lim _{s \rightarrow 0} \frac{\partial \zeta_{k}(s, 1)}{\partial s}=\zeta^{\prime}(0) \delta_{0, k-1}+\frac{1}{(k-1) !} \sum_{j=1}^{k}\left[\begin{array}{c}
k-1 \\
j
\end{array}\right] \zeta^{\prime}(-j)
$$

Here $\delta_{k, n}$ is the Kronecker delta function. Next, we sum both parts of identity (24) with respect to $k$ : 


$$
R_{n}=\sum_{k=1}^{n} \lim _{s \rightarrow 0} \frac{\partial \zeta_{k}(s, 1)}{\partial s}=\zeta^{\prime}(0)+\sum_{j=1}^{n} \zeta^{\prime}(-j) \sum_{k=j}^{n} \frac{1}{(k-1) !}\left[\begin{array}{c}
k-1 \\
j
\end{array}\right]
$$

Observing, that the inner sum can be expressed via the Stirling numbers:

$$
\sum_{k=j}^{n} \frac{1}{(k-1) !}\left[\begin{array}{c}
k-1 \\
j
\end{array}\right]=\frac{1}{(n-1) !}\left[\begin{array}{c}
n \\
j+1
\end{array}\right]
$$

we complete the proof.

Proof of Proposition 2. The derivation of (17) immediately follows from formula (14), by applying Lemma 1 and Lemma 2 :

$$
\begin{gathered}
\log \Gamma_{n}(z)=-\frac{(-1)^{n}}{(n-1) !} \sum_{j=0}^{n-1} P_{j, n}(z) \zeta^{\prime}(-j, z)+ \\
\sum_{j=0}^{n-1}(-1)^{j}\left(\begin{array}{l}
z \\
j
\end{array}\right) \frac{1}{(n-j-1) !} \sum_{k=0}^{n-j-1} \zeta^{\prime}(-k)\left[\begin{array}{l}
n-j \\
k+1
\end{array}\right]
\end{gathered}
$$

Interchanging the order of summation, we obtain

$$
\begin{gathered}
\log \Gamma_{n}(z)=\frac{(-1)^{n}}{(n-1) !} \sum_{j=0}^{n-1}\left(-P_{j, n}(z) \zeta^{\prime}(-j, z)+\right. \\
\left.\zeta^{\prime}(-k) \sum_{j=0}^{n-k}(-1)^{j}\left(\begin{array}{l}
z \\
j
\end{array}\right) \frac{(n-1) !}{(n-j-1) !}\left[\begin{array}{l}
n-j \\
k+1
\end{array}\right]\right)
\end{gathered}
$$

Finally, we note that the above inner sum is equal to representation (20) with a reversed order of summation. This completes the proof.

In the second part of this section we invert Proposition 2 and express the derivatives of the Hurwitz function in finite terms of the multiple gamma function. The problem of representing derivatives in terms of other special functions was originally addressed by Ramanujan who studied functional and asymptotic properties of this function [4]

$$
\phi_{k}(z)=\zeta^{\prime}(-k, z+1)-\zeta^{\prime}(-k) \text {. }
$$

It is unknown if Ramanujan was aware of Barnes' results regarding the multiple gamma function. 
PROPOSITION 3. The derivatives of the Hurwitz zeta function may be expressed by means of the multiple gamma function $\Gamma_{n}(z)$

$$
\zeta^{\prime}(-n, z)-\zeta^{\prime}(-n)=(-1)^{n} \sum_{k=0}^{n} k ! Q_{k, n}(z) \log \Gamma_{k+1}(z), \quad \Re(z)>0
$$

where the polynomials $Q_{k, n}(z)$ are defined by

$$
Q_{k, n}(z)=\sum_{j=k}^{n}(1-z)^{n-j}\left(\begin{array}{l}
n \\
j
\end{array}\right)\left\{\begin{array}{l}
j \\
k
\end{array}\right\}
$$

and $\left\{\begin{array}{l}j \\ k\end{array}\right\}$ are the Stirling subset numbers, defined by [16]

$$
\left\{\begin{array}{l}
n \\
k
\end{array}\right\}=k\left\{\begin{array}{c}
n-1 \\
k
\end{array}\right\}+\left\{\begin{array}{l}
n-1 \\
k-1
\end{array}\right\}, \quad\left\{\begin{array}{l}
n \\
0
\end{array}\right\}=\left\{\begin{array}{cc}
1, & n=0 \\
0, & n \neq 0
\end{array}\right.
$$

The immediate particular case of (25) is the formula for the triple gamma function:

$$
\begin{aligned}
\zeta^{\prime}(-2, z)-\zeta^{\prime}(-2) & =2 \log \Gamma_{3}(z)+(3-2 z) \log G(z) \\
& +(1-z)^{2} \log \Gamma(z), \quad \Re(z)>0
\end{aligned}
$$

For $z=0$ the polynomials $Q_{k, n}(z)$ simplify to the Stirling subset numbers:

$$
Q_{k, n}(0)=\sum_{j=k}^{n}\left(\begin{array}{l}
n \\
j
\end{array}\right)\left\{\begin{array}{l}
j \\
k
\end{array}\right\}=\left\{\begin{array}{l}
n+1 \\
k+1
\end{array}\right\}
$$

LEMMA 3. Polynomials $P_{j, n}(z)$ and $Q_{k, j}(z)$ satisfy the following discrete orthogonality relation:

$$
\sum_{j=k}^{n-1}(-1)^{j-k} Q_{k, j}(z) P_{j, n}(z)=\delta_{k, n-1}
$$

where $\delta_{k, n-1}$ is the Kronecker delta.

Proof. The proof is primarily based on the discrete orthogonality relation for the Stirling numbers:

$$
\sum_{j=0}^{n}(-1)^{m+j}\left[\begin{array}{c}
j \\
m
\end{array}\right]\left\{\begin{array}{l}
n \\
j
\end{array}\right\}=\delta_{m, n}
$$


Proof of Proposition 3. The proof readily follows from (25) by replacing $\log \Gamma_{k+1}(z)$ by (17) and then using lemma 3 .

Polynomials $Q_{k, n}(z)$ generate a great deal of new integer sequences as well as closed form representations for the existing ones. Here are two examples from the online encyclopedia of integer sequences [18].

\section{Example 1}

The sequence A021424: 1, 16, 170,1520, 12411,96096 made of coefficients by $x$ in the expansion of $1 /((1-x)(1-3 x)(1-5 x)(1-7 x))$. This sequence has a closed form representation

$$
2^{n-3} Q_{3, n}\left(\frac{1}{2}\right)=\frac{1}{48}\left(7^{n}-35^{n}+3^{n+1}-1\right)
$$

\section{Example 2}

Riordan's sequence A000554 (labeled trees of diameter 3 with $n$ nodes): $12,60,210,630,1736,4536,11430$. This sequence is generated by the coefficients of $z^{2}$ in the polynomial $2 * Q_{2, n}(1-z)$, which is also can be written as

$$
\left.\frac{\partial^{2} Q_{2, n}(1-z)}{\partial z^{2}}\right|_{z \rightarrow 0}=2\left(\begin{array}{c}
n \\
n-2
\end{array}\right)\left\{\begin{array}{c}
n-2 \\
2
\end{array}\right\}
$$

\section{Summation}

Consider the class of infinite sums

$$
\sum_{k=1}^{\infty} R(k) \log P(k)
$$

where $R(z)$ and $P(z)$ are polynomials. We will show that all such sums can be expressed in finite terms of the multiple gamma function. Clearly, (30) is a linear combination (subject to the branch cut of log) of

$$
\Phi_{N}(z)=\lim _{N \rightarrow \infty} \sum_{k=1}^{N} k^{p} \log (k+z)
$$

Thus, it is sufficient to find a closed form for (31). Expanding 


$$
k^{p}=((k+x)-x)^{p}
$$

by virtue of the binomial theorem, we get

$$
\Phi_{N}(z)=\lim _{N \rightarrow \infty} \sum_{j=0}^{p}\left(\begin{array}{l}
p \\
j
\end{array}\right)(-1)^{j} z^{p-j} F_{N}(z)
$$

where

$$
F_{N}(z)=\sum_{k=0}^{N}(k+z)^{j} \log (k+z)
$$

Assuming the analytic property of the Hurwitz zeta function, (32) can be written in the form

$$
F_{N}(z)=-\lim _{s \rightarrow \infty} \frac{d}{d s} \sum_{k=0}^{N} \frac{1}{(k+z)^{s}}=\zeta^{\prime}(-j, N+z+1)-\zeta^{\prime}(-j, z)
$$

and therefore

$$
\begin{aligned}
\Phi_{N}(z)= & \lim _{N \rightarrow \infty} \sum_{j=0}^{p}\left(\begin{array}{l}
p \\
j
\end{array}\right)(-1)^{j+1} z^{p-j} \\
& \left(\zeta^{\prime}(-j, N+z+1)-\zeta^{\prime}(-j, z)\right)
\end{aligned}
$$

Further, we replace the first cumulant $\zeta^{\prime}(-j, z)$ by a linear combination of the multiple gamma function (see Proposition 3), and the second cumulant $\zeta^{\prime}(-j, N+z+1)$ by asymptotic (11). When the original series (30) converges, the $N$-dependent term will vanish as $N \rightarrow \infty$. However, if series (30) diverges, formula (33) combined with (111) will provide a zeta regularization of (30) in the Hadamard sense.

In order to make this idea clear, we will consider a few examples.

\subsection{DilcheR's SUM}

In 17, Dilcher introduced a particular generalization of the Euler gamma function $\Gamma(x)$ which is related to the Stieltjes constants $\gamma_{k}$, as $\Gamma(x)$ is related to the Euler $\gamma$ constant. The Stieltjes constants $\gamma_{k}$ are defined as coefficients in the Laurent expansion of the Riemann zeta function $\zeta(s)$ at the simple pole $s=1$ :

$$
\zeta(s)=\frac{1}{s-1}+\sum_{k=0}^{\infty} \frac{(-1)^{k}}{k !} \gamma_{k}(s-1)^{k}
$$


The Euler $\gamma$ constant is a particular case of the Stieltjes constants $\gamma_{0}=\gamma$. Dilcher derived a few basic properties of the generalized gamma function as well as its asymptotic expansion when $z \rightarrow \infty$. The following infinite series appears as a constant term in the asymptotic expansion:

$$
D_{k}=\sum_{j=1}^{\infty} \log ^{k}\left(j+\frac{1}{2}\right)-2 \log ^{k} j+\log ^{k}\left(j-\frac{1}{2}\right)
$$

For $k=1$ the sum (34) simplifies to $D_{1}=\log (2 / \pi)$. For other values of $k>1$, the closed form was unknown. In this section, we apply the technique of the multiple gamma function and evaluate the logarithmic series (34) in closed form.

We begin with a finite sum

$$
D_{k}=\lim _{N \rightarrow \infty} \sum_{j=1}^{N} \log ^{k}\left(j+\frac{1}{2}\right)-2 \log ^{k} j+\log ^{k}\left(j-\frac{1}{2}\right)
$$

and then rewrite it in terms of the Hurwitz function. The following chain of operations is valid (assuming the analytic property of the Hurwitz function)

$$
\begin{gathered}
(-1)^{k} \sum_{j=1}^{N} \log ^{k}(j+c)=\lim _{s \rightarrow 0} \frac{\partial^{k}}{\partial s^{k}} \sum_{j=1}^{N} \frac{1}{(j+c)^{s}}= \\
\lim _{s \rightarrow 0} \frac{\partial^{k}}{\partial s^{k}}\left(\sum_{j=1}^{\infty} \frac{1}{(j+c)^{s}}-\sum_{j=N+1}^{\infty} \frac{1}{(j+c)^{s}}\right)= \\
\zeta^{k}(0, c+1)-\zeta^{k}(0, c+N+1)
\end{gathered}
$$

Applying this to (35), yields

$$
\begin{aligned}
(-1)^{k} D_{k}= & -2 \zeta^{k}(0,1)+\zeta^{k}\left(0, \frac{1}{2}\right)+\zeta^{k}\left(0, \frac{3}{2}\right)+ \\
& \lim _{N \rightarrow \infty}\left(2 \zeta^{k}(0, N+1)-\zeta^{k}\left(0, N+\frac{1}{2}\right)-\zeta^{k}\left(0, N+\frac{3}{2}\right)\right)
\end{aligned}
$$

Now we use the asymptotic expansion of $\zeta^{k}(0, N), N \rightarrow \infty$. From the Hermite integral (2) it follows that the dominant asymptotic term of $\zeta^{k}(0, N)$ comes from the first two terms

$$
\zeta(s, z)=\frac{z^{-s}}{2}+\frac{z^{1-s}}{s-1}+\ldots
$$

This leads to the following asymptotic expansion $N \rightarrow \infty$ 


$$
\begin{aligned}
\zeta^{k}(0, N)= & N \sum_{j=0}^{k}(-1)^{j}(k-j+1)_{j} \log ^{k-j+1} N+ \\
& \frac{(-1)^{k}}{2} \log ^{k} N+O\left(\frac{1}{N}\right),
\end{aligned}
$$

where $(k-j+1)_{j}$ is the Pochhammer symbol. Thus, to prove that the limiting part in (37) vanishes to zero, it is sufficient to show that

$$
\begin{gathered}
\lim _{N \rightarrow \infty}\left[2(N+1) \log ^{j}(N+1)-\right. \\
\left.\left(N+\frac{1}{2}\right) \log ^{j}\left(N+\frac{1}{2}\right)-\left(N+\frac{3}{2}\right) \log ^{j}\left(N+\frac{3}{2}\right)\right]=0
\end{gathered}
$$

for any $j=1,2, \ldots, k+1$. This follows immediately from the fact that

$$
\log (N+c)=\log N+\frac{c}{N}+O\left(\frac{1}{N^{2}}\right), \quad N \rightarrow \infty
$$

and the sum of coefficients by $\log ^{k-j+1}(N)$ in (39) is zero.

Therefore, we have

$$
D_{k}=(-1)^{k}\left(-\log ^{k} 2-2 \zeta^{k}(0)+2 \zeta^{k}\left(0, \frac{1}{2}\right)\right)
$$

Note, $\zeta^{k}\left(0, \frac{1}{2}\right)$ in the right hand-side of (401) is a linear combination of $\zeta^{k}(0)$ :

$$
\zeta^{k}\left(0, \frac{1}{2}\right)=-\frac{1}{2} \log ^{k} 2+\sum_{j=1}^{k-1}\left(\begin{array}{l}
k \\
j
\end{array}\right) \zeta^{k-j}(0) \log ^{j} 2
$$

Combining this with (40), yields

PROPOSITION 4. The infinite series (34) can be expressed in finite terms of the derivatives of the Riemann zeta function

$$
\frac{(-1)^{k}}{2} D_{k}=-\log ^{k} 2-\zeta^{k}(0)+\sum_{j=1}^{k-1}\left(\begin{array}{l}
k \\
j
\end{array}\right) \zeta^{k-j}(0) \log ^{j} 2
$$

In particular, for $k=2$ and $k=3$ we have

$$
\begin{aligned}
D_{2} & =\sum_{j=1}^{\infty} \log ^{2}\left(j+\frac{1}{2}\right)-2 \log ^{2} j+\log ^{2}\left(j-\frac{1}{2}\right) \\
& =\frac{\pi^{2}}{12}+\log ^{2} \pi-3 \log ^{2} 2-\gamma^{2}-2 \gamma_{1}
\end{aligned}
$$




$$
D_{3}=3 D_{2} \log 2+2 \zeta^{\prime \prime \prime}(0)+9 \log (2 \pi) \log ^{2} 2+\log ^{3} 4
$$

respectively.

Note that by the functional equation

$$
\zeta(s)=2^{s} \pi^{s-1} \Gamma(1-s) \sin \left(\frac{\pi s}{2}\right) \zeta(1-s), \quad \Re(s) \neq 1
$$

the Riemann zeta function admits a meromorphic continuation to the entire complex plane. Differentiating this with respect to $s$ and setting $s=0$, allows us to express derivatives $\zeta^{k}(0)$ in finite terms of the Stieltjes constants (see [2] for a closed form evaluation). Therefore, formulas like (42) and (43) can be used for numeric computation of $\zeta^{k}(0)$ as well as the Stieljes constants $\gamma_{k}$.

\subsection{MelzaK's Product}

Let us consider the infinite product

$$
\lim _{N \rightarrow \infty} \prod_{k=1}^{2 N}\left(1+\frac{2 x}{k}\right)^{-k(-1)^{k}}
$$

and evaluate it in closed form. Originally, this product was computed by Melzak [12] in a particular case when $x=1$

$$
\lim _{N \rightarrow \infty} \prod_{k=1}^{2 N}\left(1+\frac{2}{k}\right)^{-k(-1)^{k}}=\frac{\pi}{2 e}
$$

Later on, P. Borwein and W. Dykshoorn [5] generalized (45) and computed it in terms of Bendersky's function, defined by (see [26])

$$
1^{1} 2^{2} \ldots n^{n}=\Gamma_{1}(n+1)
$$

which is closely related to the Barnes function $G$

$$
\Gamma_{1}(n+1)=\frac{n !^{n}}{G(n+1)}
$$

Note that (44) can be written in an alternative form by changing the product limit from $2 N$ to $2 N+1$ :

$$
\lim _{N \rightarrow \infty} \prod_{k=1}^{2 N+1}\left(1+\frac{2 x}{k}\right)^{-k(-1)^{k}}=e^{2 x} \lim _{N \rightarrow \infty} \prod_{k=1}^{2 N}\left(1+\frac{2 x}{k}\right)^{-k(-1)^{k}}
$$


In this section, using the multiple gamma function technique, we reevaluate product (44), derive its few particular cases in terms of known constants and then generalize it to

$$
\lim _{N \rightarrow \infty} \prod_{k=1}^{2 N}\left(1-\frac{4 x^{2}}{k^{2}}\right)^{-k^{2}(-1)^{k}}
$$

PROPOSITION 5. The following identity holds for $\Re(x)>-\frac{1}{2}$

$$
\lim _{N \rightarrow \infty} \prod_{k=1}^{2 N}\left(1+\frac{2 x}{k}\right)^{-k(-1)^{k}}=\frac{e^{-x} \Gamma\left(x+\frac{1}{2}\right)}{\Gamma\left(\frac{1}{2}\right)}\left(\frac{G\left(x+\frac{1}{2}\right)}{G(x+1) G\left(\frac{1}{2}\right)}\right)^{2}
$$

Proof. We first convert this product into a finite sum by applying the logarithm to it:

$$
\begin{aligned}
\lim _{N \rightarrow \infty} \prod_{k=1}^{2 N}\left(1+\frac{2 x}{k}\right)^{-k(-1)^{k}} & = \\
& \quad \exp \left(\lim _{N \rightarrow \infty} \sum_{k=1}^{2 N}(-1)^{k} k(\log k-\log (k+2 x))\right)
\end{aligned}
$$

In the next step we split the finite sum in the right hand-side of (47) into three sums:

$$
\begin{array}{r}
\sum_{k=1}^{2 N}(-1)^{k} k(\log k-\log (k+2 x))=2 x \sum_{k=1}^{2 N}(-1)^{k} \log (k+2 x)+ \\
\sum_{k=1}^{2 N}(-1)^{k} k \log k-\sum_{k=1}^{2 N}(-1)^{k}(k+2 x) \log (k+2 x)
\end{array}
$$

and evaluate each of them in terms of the Hurwitz function. For arbitrary $c$ such that $\Re(c)>0$, we find

$$
\begin{array}{r}
\sum_{k=1}^{2 N}(-1)^{k}(k+c) \log (k+c)=-\lim _{s \rightarrow-1} \frac{\partial}{\partial s} \sum_{k=1}^{2 N} \frac{(-1)^{k}}{(k+c)^{s}}= \\
N \log 2+2 \zeta^{\prime}\left(-1, \frac{c+1}{2}\right)-2 \zeta^{\prime}\left(-1, \frac{c+2}{2}\right)+ \\
2 \zeta^{\prime}\left(-1, \frac{c+2}{2}+N\right)-2 \zeta^{\prime}\left(-1, \frac{c+1}{2}+N\right)
\end{array}
$$


and

$$
\begin{array}{r}
\sum_{k=1}^{2 N}(-1)^{k} \log (k+c)=\zeta^{\prime}\left(0, \frac{c+1}{2}\right)-\zeta^{\prime}\left(0, \frac{c+2}{2}\right)+ \\
\zeta^{\prime}\left(0, \frac{c+2}{2}+N\right)-\zeta^{\prime}\left(0, \frac{c+1}{2}+N\right)
\end{array}
$$

where derivatives $\zeta^{\prime}(-\lambda, z)$ are understood by (8) . Now we use Proposition 1 , in particular asymptotic expansions (13), to get

$$
\begin{array}{r}
\lim _{N \rightarrow \infty} \sum_{k=1}^{2 N}(-1)^{k} k(\log k-\log (k+2 x))= \\
2 x \log \Gamma\left(x+\frac{1}{2}\right)-2 x \log \Gamma(x+1)- \\
2 \zeta^{\prime}\left(-1, x+\frac{1}{2}\right)+2 \zeta^{\prime}(-1, x+1)- \\
\frac{\log 2}{12}-x-3 \zeta^{\prime}(-1)
\end{array}
$$

In view of Proposition 3 with $n=1$, we convert the derivatives of the Hurwitz function to the Barnes function:

$$
\begin{gathered}
\zeta^{\prime}(-1, z)=-\log G(z+1)+z \log \Gamma(z)+\zeta^{\prime}(-1) \\
3 \zeta^{\prime}(-1)=-\frac{\log 2}{12}+\frac{\log \pi}{2}+2 \log G\left(\frac{1}{2}\right)
\end{gathered}
$$

Combining these with (48), after some algebraic manipulations, leads to (46).

Here are a few particular cases of (46):

$$
\begin{gathered}
\lim _{N \rightarrow \infty} \prod_{k=1}^{2 N}\left(1+\frac{1}{k}\right)^{-k(-1)^{k}}=\frac{A^{6}}{e \sqrt{\pi} 2^{1 / 6}} \\
\lim _{N \rightarrow \infty} \prod_{k=1}^{2 N}\left(1+\frac{4}{k}\right)^{-k(-1)^{k}}=\frac{3 \pi^{2}}{16 e^{2}} \\
\lim _{N \rightarrow \infty} \prod_{k=1}^{2 N}\left(1-\frac{1}{2 k}\right)^{-k(-1)^{k}}=\frac{A^{3} e^{-\mathbf{G} / \pi} \sqrt{\pi} 2^{1 / 6}}{\Gamma\left(\frac{1}{4}\right)}
\end{gathered}
$$

where $A$ is the Glaisher-Kinkelin constant and $\mathbf{G}$ is Catalan's constant defined by 


$$
\begin{gathered}
\log A=\frac{1}{12}-\zeta^{\prime}(-1) \\
\mathbf{G}=\sum_{k=0}^{\infty} \frac{(-1)^{k}}{(2 k+1)^{2}}
\end{gathered}
$$

respectively.

The Melzak product can be further generalized. Here is one of such formulas

PROPOSITION 6. The following identity holds for $\Re(x)>-\frac{1}{2}$ :

$$
\begin{aligned}
& \lim _{N \rightarrow \infty} \prod_{k=1}^{2 N}\left(1-\frac{4 x^{2}}{k^{2}}\right)^{-k^{2}(-1)^{k}}=\frac{\cos (\pi x)}{\pi} \exp \left(2 x^{2}+\frac{7 \zeta(3)}{2 \pi^{2}}\right) \\
& (G(1+x) G(1-x))^{4}\left(\frac{\Gamma_{3}\left(\frac{3}{2}-x\right) \Gamma_{3}\left(\frac{3}{2}+x\right)}{\Gamma_{3}(1-x) \Gamma_{3}(1+x)}\right)^{8}
\end{aligned}
$$

We skip the proof of this proposition, since it could be done in the same manner as in Proposition 5.

CORROLARY 1. Identity (52) can be further simplified to

$$
\begin{gathered}
\lim _{N \rightarrow \infty} \prod_{k=1}^{2 N}\left(1-\frac{4 x^{2}}{k^{2}}\right)^{-k^{2}(-1)^{k}}= \\
\tan (\pi x)^{-4 x^{2}} \exp \left[2 x^{2}(1+\pi i)+\frac{7 \zeta(3)}{2 \pi^{2}}+\right. \\
\left.\frac{4 i x}{\pi}\left(\operatorname{Li}_{2}(\omega)-\operatorname{Li}_{2}(-\omega)\right)-\frac{2}{\pi^{2}}\left(\operatorname{Li}_{3}(\omega)-\operatorname{Li}_{3}(-\omega)\right)\right]
\end{gathered}
$$

where $\omega=\exp (2 \pi i x)$ and $\operatorname{Li}_{k}(\omega)$ is the polylogarithm, defined by

$$
\operatorname{Li}_{k}(\omega)=\sum_{k=0}^{\infty} \frac{\omega^{k}}{n^{k}}, \quad k>1,|\omega| \leq 1
$$

Proof. The proof follows straightforwardly from (52) upon employing the reflexion formulas for the multiple gamma function:

$$
\log \frac{G(1+z)}{G(1-z)}=z \log \left(\frac{\pi}{\sin \pi z}\right)-\frac{\pi i}{2} B_{2}(z)+\frac{i}{2 \pi} \operatorname{Li}_{2}\left(e^{2 \pi i z}\right)
$$




$$
\begin{gathered}
2 \log \left(\Gamma_{3}(1+z) \Gamma_{3}(1-z)\right)+\log (G(1+z) G(1-z))= \\
z^{2} \log \left(\frac{\pi}{\sin \pi z}\right)-\pi i z B_{2}(z)+\frac{\pi i}{3} B_{3}(z)+ \\
\frac{i z}{\pi} \operatorname{Li}_{2}\left(e^{2 \pi i z}\right)-\frac{1}{2 \pi^{2}} \operatorname{Li}_{3}\left(e^{2 \pi i z}\right)+\frac{\zeta(3)}{2 \pi^{2}}
\end{gathered}
$$

where $B_{k}(z)$ are the Bernoulli polynomials.

Proposition 6 yields the following particular cases:

$$
\begin{gathered}
\lim _{N \rightarrow \infty} \prod_{k=1}^{2 N}\left(1-\frac{1}{4 k^{2}}\right)^{-k^{2}(-1)^{k}}=\exp \left(\frac{1}{8}-\frac{2 G}{\pi}+\frac{7 \zeta(3)}{2 \pi^{2}}\right) \\
\lim _{N \rightarrow \infty} \prod_{k=2}^{2 N}\left(1-\frac{1}{k^{2}}\right)^{-k^{2}(-1)^{k}}=\frac{\pi}{4} \exp \left(\frac{1}{2}+\frac{7 \zeta(3)}{\pi^{2}}\right)
\end{gathered}
$$

Formula (54) follows from (53) with $x=\frac{1}{4}, \omega=i$ and taking into account

$$
\begin{aligned}
& \mathrm{Li}_{2}(i)-\mathrm{Li}_{2}(-i)=2 i \mathbf{G} \\
& \mathrm{Li}_{3}(i)-\mathrm{Li}_{3}(-i)=\frac{i \pi^{3}}{16}
\end{aligned}
$$

Similarly, (55) follows from (53) with $x=\frac{1}{2}, \omega=-1$ and

$$
\begin{gathered}
\operatorname{Li}_{2}(1)-\mathrm{Li}_{2}(-1)=\frac{\pi^{2}}{4} \\
\mathrm{Li}_{3}(1)-\mathrm{Li}_{3}(-1)=\frac{7 \zeta(3)}{4} \\
\lim _{x \rightarrow \frac{1}{2}} \frac{\tan ^{-4 x^{2}}(\pi x)}{1-4 x^{2}}=\frac{\pi}{4}
\end{gathered}
$$

\section{Acknowledgements}

This work was supported by grant CCR-0204003 from the National Science Foundation. 


\section{References}

1. V. S. Adamchik, H. M. Srivastava, Some series of the zeta and related functions, Analysis (1998) 131-144.

2. T. M. Apostol, Formulas for higher derivatives of the Riemann zeta function, Math. Comp. 44 (1985) 223-232.

3. L. Bendersky, Sur la fonction gamma généralisée, Acta Math. 61 (1933) 263322 .

4. B. Berndt, Ramanujan's Notebooks, Part I, Springer-Verlag, New York, 1985.

5. P. Borwein, W. Dykshoorn, An Interesting Infinite Product, J. Math. Anal. and Appl. 179 (1993) 203-207.

6. J. Choi, H. M. Srivastava, V.S.Adamchik, Multiple Gamma and related functions, Appl. Math. and Comp. 134(2003) 515-533.

7. K. Dilcher, On generalized gamma functions related to the Laurent coefficients of the Riemann zeta function, Aequationes Math. 179 (1994) 55-85

8. O. Espinosa, V. Moll, On some integrals involving the Hurwitz zeta function, The Ramanujan Journal. 6 (2002) 159-188.

9. J. P. Keating, N. C. Snaith, Random matrix theory and $\zeta\left(\frac{1}{2}+i t\right)$, Commun. Math. Phys. 214 (2000) 57-89.

10. J. P. Keating, N. C. Snaith, Random matrix theory and $L$-functions at $s=\frac{1}{2}$, Commun. Math. Phys. 214 (2000) 91-110.

11. W. Magnus, F. Oberhettinger, R.P. Soni, Formulas and theorems for the special functions of mathematical physics Springer, Berlin, 1966.

12. Z. A. Melzak, Infinite Products for $\pi e$ and $\pi / e$, MAA Monthly $68 \quad$ (1961) 39-41.

13. D. S. Mitrinovic, Sur une classe de nombres relis aux nombres de Stirling, $C$. R. Acad. Sci. Paris 252 (1961) 2354-2356.

14. H. L. Montgomery, The pair correlation of the zeta function, Proc. Symp. Pure Math. 24 (1973) 181-193.

15. A. M. Odlyzko, The $10^{22}$-nd zero of the Riemann zeta function, Dynamical, Spectral, and Arithmetic Zeta Functions; M. van Frankenhuysen and M. L. Lapidus, eds., Amer. Math. Soc., Contemporary Math. series, no. 290 (2001) 139-144.

16. K. N. Rosen and et.., Handbook of Discrete and Combinatorial Mathematics (CRC Press, New York, 2000).

17. P. Sarnak, Quantum chaos, symmetry and zeta functions, Curr. Dev. Math. (1997) 84-115.

18. N. Sloan, The online encyclopedia of integer sequences http://www.research.att.com njas/sequences/

19. I. Vardi, Determinants of Laplacians and multipe gamma functions, SIAM J. Math. Anal. 19(1988)493-507.

20. E. T. Whittaker, G. N. Watson, A course of Modern Analysis: An Introduction to the General Theory of Infinite Processes and of Analytic Functions; With an Account of the Principal Transcendental Functions, Fourth Ed. (Cambridge University Press, Cambridge, 1963). 